\title{
Development of a position-specific isotopic proxy for photorespiration
}

Elise B. Wilkes ${ }^{1 *}$, AleX L. Sessions ${ }^{1}$, JOHN M. EILER ${ }^{1}$

${ }^{1}$ Division of Geological and Planetary Sciences, California Institute of Technology, Pasadena, California, USA

(*ebwilkes@caltech.edu)

Photorespiration is a wasteful metabolic process that occurs when the initial enzyme of the Calvin cycle, Rubisco, fixes $\mathrm{O}_{2}$ instead of $\mathrm{CO}_{2}$. This process reduces the efficiency of photosynthesis with significant implications for biogeochemical cycling and crop production [1]. The extent of photorespiration is a function of physiology, $\mathrm{CO}_{2} / \mathrm{O}_{2}$ ratio, and temperature. Accurately understanding photorespiratory fluxes in different organisms and environments is critical for predicting how the biosphere will respond to climate change.

We hypothesize that intramolecular variations in stable carbon isotope ratios $\left({ }^{13} \mathrm{C} /{ }^{12} \mathrm{C}\right)$ in the amino acid, serine, may serve as a proxy for photorespiratory fluxes. The proxy rationale is derived from knowledge of serine's biosynthesis and predicted isotopic fractionations [2,3]. Such a proxy holds the potential to integrate spatial and temporal heterogeneity of photorespiration in ecosystems. Here we present our first step towards this goal: method development enabling the position-specific isotope analysis of serine by GC-Orbitrap mass spectrometry. Serine molecules are isolated by gas chromatography, fragmented in an electron ionization source, and the resulting fragments are analyzed for long periods of time at high mass resolution. Because fragments sample different combinations of atomic positions from the parent molecule, this measurement provides multiple constraints on the spatial distribution of isotopes within the original molecule [4]. We will also discuss ${ }^{13} \mathrm{C} /{ }^{12} \mathrm{C}$ distributions obtained in a pilot study of Arabidopsis thaliana exposed to different $\mathrm{CO}_{2} / \mathrm{O}_{2}$ ratios and the resulting prospects for a photorespiratory proxy.

[1] Walker BJ, et al. (2016) Annual Review of Plant Biology 67, 107-129. [2] Tcherkez G, et al. (2011) Trends in Plant Science 16, 499-506. [3] Tcherkez G (2006) Functional Plant Biology 10, 911-920. [4] Eiler J, et al. (2017) International Journal of Mass Spectrometry 422, 126-142. 